\title{
Association between serum levels and pentanucleotide polymorphism in the sex hormone binding globulin gene and cardiovascular risk factors in females with polycystic ovary syndrome
}

\author{
DINKA PAVICIC BALDANI ${ }^{1}$, LANA SKRGATIC ${ }^{1}$, JASMINA ZIVA CERNE ${ }^{2}$, \\ SASA KRALIK OGUIC ${ }^{3}$, BLAZ MATIJA GERSAK ${ }^{4}$ and KSENIJA GERSAK ${ }^{2,4}$ \\ ${ }^{1}$ Department of Obstetrics and Gynecology, University of Zagreb Medical School, Division of Human Reproduction, \\ University Medical Centre Zagreb, 10000 Zagreb, Croatia; ${ }^{2}$ Department of Obstetrics and Gynecology, \\ University Medical Centre Ljubljana, Ljubljana SI-1000, Slovenia; ${ }^{3}$ Clinical Institute of Laboratory Diagnostics, \\ University Medical Centre Zagreb, 10000 Zagreb, Croatia; ${ }^{4}$ Faculty of Medicine, \\ University of Ljubljana, Ljubljana SI-1104, Slovenia
}

Received January 24, 2014; Accepted November 3, 2014

DOI: $10.3892 / \mathrm{mmr} .2014 .3117$

\begin{abstract}
The objective of the present study was to evaluate the influence of TAAAA repeat allele length on the levels of serum sex hormone binding globulin (SHBG) and cardiovascular risk factors in patients with polycystic ovary syndrome (PCOS). The study included 91 females with PCOS and 99 healthy controls. Phenotypic hyperandrogenism, body mass index and waist-to-hip ratio (WHR) were recorded. Hormonal profiles, fasting insulin and glucose levels, lipid profiles and C-reactive protein (CRP) levels were measured. Genotyping of TAAAA repeat polymorphisms in the $S H B G$ gene was performed. No significant difference was found in the frequency and distribution of TAAAA repeat alleles between PCOS patients and controls $(\mathrm{P}=0.739)$. In PCOS patients, SHBG levels were inversely correlated with serum C-reactive protein (CRP) levels $(\mathrm{R}=-0.489, \mathrm{P}<0.001)$. PCOS patients with long TAAAA repeat alleles had significantly lower serum SHBG and free testosterone levels, yet higher CRP levels than patients with short allele repeats. A multiple linear regression model using the number of TAAAA repeats, waist-to-hip ratio, a homeostatic model assessment of insulin resistance and age as independent predictors explained $44.8 \%$ of the variability in serum SHBG levels. In this model, TAAAA repeat polymorphism was found to be the only reliable predictor of serum SHBG levels $(\mathrm{P}<0.001)$. In conclusion, the TAAAA repeat polymorphism was shown
\end{abstract}

Correspondence to: Professor Ksenija Gersak, Department of Obstetrics and Gynecology, University Medical Centre Ljubljana, 3 Slajmerjeva, Ljubljana SI-1000, Slovenia

E-mail: ksenija.gersak@mf.uni-lj.si

Key words: sex hormone binding globulin, C-reactive protein, TAAAA repeat polymorphism, polycystic ovary syndrome to not be a major determinant of the PCOS status, although it influenced serum SHBG levels in females with PCOS. A strong independent association existed between serum SHBG and CRP levels. CRP is an established risk factor of cardiovascular disease and a marker of low-grade inflammation, typical of atherogenesis. This may be one of the pathways by which low SHBG levels affect cardiovascular risk.

\section{Introduction}

Polycystic ovary syndrome (PCOS) is the most common endocrine disorder in females of reproductive age. It is characterized by oligomenorrhea, hyperandrogenemia (or hyperandrogenism) and polycystic ovaries on an ultrasound scan, with two of these criteria required to be fulfilled to diagnose PCOS $(1,2)$. Obesity, accumulation of abdominal fat and insulin resistance often accompany PCOS, although the occurrence of these parameters varies between different ethnic groups (3).

It has been reported that females with PCOS have significantly increased levels of C-reactive protein (CRP) (4), which is a marker of low-grade chronic inflammation and a predictor of cardiovascular diseases (CVD). While it has been discussed whether obesity, and not the PCOS status per se, is the major determinant of circulating inflammatory marker levels $(4,5)$, a recent meta-analysis revealed that $\mathrm{CRP}$ is the most reliable circulating marker of chronic low-grade inflammation in PCOS, independent of obesity (6). Since a large number of females with PCOS develop metabolic abnormalities at an early age, making them strong candidates for early-onset CVD $(1,2,7)$, there is an emerging necessity to discover the mechanism linking PCOS with CVD. It has been suggested that increased androgenicity, characterized by high testosterone and low sex hormone binding globulin (SHBG) levels, is associated with an adverse CVD risk factor profile in females (8).

In PCOS patients, serum sex hormone binding globulin (SHBG) levels are generally low, contributing to increased 
tissue androgen bioavailability (9). Low SHBG has been shown to be an independent cardiovascular risk factor, in addition to a risk factor for developing type 2 diabetes $(2,10-13)$ and metabolic syndromes (8). While it is well established that the hepatic synthesis of SHBG is subject to the influence of hormonal, metabolic and nutritional factors, there is also evidence that SHBG production is genetically determined (14). The TAAAA pentanucleotide polymorphism occurs in the promoter region of the SHBG gene and has been reported to affect its transcriptional activity in vitro (15). A strong association has been observed between the TAAAA functional polymorphism and circulating SHBG levels in females with PCOS from different ethnic backgrounds (16-19,20).

Longer TAAAA repeats of the SHBG gene have been shown to be associated with the severity of coronary artery disease in postmenopausal females undergoing coronary angiography (20). It has been recently reported that longer alleles at the promoter of the SHBG gene are associated with early markers of atherosclerosis in apparently healthy females (21). The aim of the present study was to evaluate the influence of the TAAAA repeat allele length on serum SHBG levels and on cardiovascular risk factors in a Croatian population of PCOS patients.

\section{Subjects and methods}

Study subjects. A total of 91 females who fulfilled the criteria for PCOS were enroled in the present study. The diagnosis of PCOS was made according to the Rotterdam consensus criteria, which are based on the presence of two out of three traits, including oligo- and/or anovulation, clinical and/or biochemical symptoms of hyperandrogenism and polycystic ovaries on an ultrasound scan $(1,2)$. Other endocrinopathies and associated disorders were ruled out by measuring basal serum 17-hydroxyprogesterone (17-OHP), prolactin (PRL) and thyroid-stimulating hormone (TSH) levels. Menstrual irregularities were defined as the presence of ameno- or oligomenorrhea. Hyperandrogenism was assessed as the presence of hirsutism and/or acne and/or elevated androgen levels [serum total testosterone $(\mathrm{TT})>2.5 \mathrm{nmol} / \mathrm{l}$ or free testosterone (FT) $>30 \mathrm{pmol} / \mathrm{l}]$. Hirsutism was defined as a Ferriman-Gallwey score $>8(\mathrm{FG}>8)(22)$.

The control group consisted of 99 healthy volunteers prior to entering an in vitro fertilization programme for male factor infertility. They were free of menstrual cycle irregularities, clinical or biochemical hyperandrogenism, PCO according to an ultrasound scan, and had no history of endocrine or autoimmune disorders or surgery in the pelvic region.

Information on the family history of coronary artery disease (CAD), hypertension, dyslipidemia, diabetes, personal history of hypertension, childhood obesity and diabetes, age at pubarche and menarche was obtained from each patient during a medical interview. All patients were observed in the early follicular phase of the menstrual cycle (day 3-5) or randomly in amenorrhoeic patients. The body mass index (BMI) was calculated as weight $/$ height ${ }^{2}\left(\mathrm{~kg} / \mathrm{m}^{2}\right)$. The waist-to-hip ratio (WHR) was calculated by dividing the waist circumference by the hip circumference. For the diagnosis of PCO, according to the Rotterdam criteria, a transvaginal ultrasound scan was performed $(1,2)$. The patients were without any hormonal medication or hormonal contraceptives for $\geq 6$ months prior to entering the study. Blood samples were drawn for the measurement of luteinizing hormone ( $\mathrm{LH})$, follicle-stimulating hormone (FSH), TT, SHBG, dehydroepiandrosteronesulfate (DHEAS), androstenedione (AN), fasting serum glucose, fasting insulin, total cholesterol, high-density cholesterol (HDL), triglyceride and CRP levels. FT was calculated from TT and SHBG as previously described (23) using a web-based calculator (http://www. issam.ch/freetesto.htm). Insulin resistance (IR) was quantified using the homeostatic model assessment of IR (HOMA-IR): [fasting insulin (mU/l) x fasting glucose $(\mathrm{mmol} / \mathrm{l})] / 22.5$. The LDL cholesterol concentration was calculated mathematically from the total cholesterol, triglyceride and HDL cholesterol concentrations using the Friedewald's formula (24): LDL cholesterol $(\mathrm{mmol} / \mathrm{l})=$ total cholesterol $(\mathrm{mmol} / \mathrm{l})-\mathrm{HDL}$ cholesterol (mmol/l) - triglycerides $(\mathrm{mmol} / \mathrm{l}) / 2.2$.

The present study was approved by the Ethics Committee of the University of Zagreb Medical School (study protocol no. 04-1116-2006). Informed written consent was obtained from all females enrolled in the study.

Hormone assays. Serum LH, FSH, SHBG, TT, DHEAS and AN levels were measured successively. Serum LH, FSH, TSH, prolactin and TT concentrations were determined by chemiluminescent immunometric assays using the Vitros $\mathrm{LH}, \mathrm{FSH}$, TSH, prolactine, and TT reagent packs, respectively (Ortho Clinical Diagnostics, Johnson\&Johnson, Rochester, NY, USA). Serum SHBG, DHEAS and AN levels were measured using chemiluminescent immunometric assays (SHBG-Immulite, DHEAS-Immulite, and Androstenedione-Immulite, respectively; Siemens Healthcare Diagnostics, Inc., Deerfield, IL, USA). The concentration of 17-OH-progesterone was determined by a solid-phase enzyme-linked immunosorbent assay (17- $\alpha-\mathrm{OHP}$ DRG ELISA kit) based on the principle of competitive binding (DRG-diagnostics, Marburg, Germany). The intra-assay and inter-assay coefficients of variation ranged between 1.5 and $7.9 \%$. The plasma glucose levels were determined using the ultraviolet-photometric hexokinase method, and the serum insulin levels with a chemiluminescent immunometric assay using Insulin-Immulite (Siemens Healthcare Diagnostics, Inc., New York, NY, USA). Total cholesterol and triglycerides were measured with a routine enzymatic photometric assay using Siemens CHOL and Siemens TGL respectively, on the Siemens Dimension Xpand clinical chemistry system (Siemens Healthcare, Erlangen, Germany). HDL cholesterol was determined following the precipitation of VLDL and LDL with dextran sulphate and $\mathrm{MgCl}_{2}$ (Merck KGaA, Darmstadt, Germany). CRP was determined by a particle enhanced turbidimetric immunoassay technique on the Siemens Dimension Xpand clinical chemistry system with Siemens RCRP reagent (Siemens Healthcare Diagnostics Inc.). Synthetic particles coated with antibody to C-reactive protein (CRP) aggregate in the presence of CRP in the sample. The increase in turbidity which accompanies aggregation was proportional to the CRP concentration. The test sensitivity was $0.1 \mathrm{mg} / \mathrm{l}$. Whole blood samples were obtained for genetic analyses.

Genotype analysis. Genomic DNA was isolated from whole blood using a FlexiGene kit (Qiagen GmbH, Hilden, Germany) following the manufacturer's instructions. Genotyping for the $S H B G$ (TAAAA)n polymorphismwasperformed by polymerase 
Table I. Clinical and hormonal parameters in PCOS patients and healthy controls.

\begin{tabular}{|c|c|c|c|}
\hline Variable & $\operatorname{PCOS}(n=91)$ & Control $(n=99)$ & P-value ${ }^{a}$ \\
\hline Age (years) & $28.3 \pm 5.7$ & $31.3 \pm 4.8$ & $<0.001$ \\
\hline BMI $\left(\mathrm{kg} / \mathrm{m}^{2}\right)$ & $24.1 \pm 4.7$ & $23.3 \pm 4.1$ & 0.183 \\
\hline BMI <25 (kg/m²) & $69.2 \%$ & $80.8 \%$ & 0.065 \\
\hline WHR & $0.78 \pm 0.1$ & $0.79 \pm 0.1$ & 0.941 \\
\hline Family history of CAD & $27.5 \%$ & NA & NA \\
\hline Hypertension in family & $33.0 \%$ & NA & NA \\
\hline Dyslipidemia in family & $7.7 \%$ & NA & NA \\
\hline Diabetes in family & $36.3 \%$ & NA & NA \\
\hline History of hypertension & $2.2 \%$ & NA & NA \\
\hline Childhood obesity & $42.9 \%$ & NA & NA \\
\hline Diabetes & $0.0 \%$ & NA & NA \\
\hline FSH (IU/l) & $3.2 \pm 0.9$ & $5.5 \pm 1.6$ & $<0.001$ \\
\hline LH (IU/l) & $7.9 \pm 5.2$ & $3.9 \pm 1.1$ & $<0.001$ \\
\hline TT (nmol/l) & $2.1 \pm 0.9$ & $1.2 \pm 0.5$ & $<0.001$ \\
\hline FT (pmol/l) & $39.3 \pm 23.6$ & $14.3 \pm 6.8$ & $<0.001$ \\
\hline Androstenedione (nmol/l) & $11.0 \pm 4.8$ & $7.2 \pm 2.8$ & $<0.001$ \\
\hline DHEAS $(\mu \mathrm{mol} / \mathrm{l})$ & $6.3 \pm 2.1$ & $4.3 \pm 2.0$ & $<0.001$ \\
\hline SHBG (nmol/l) & $38.4 \pm 19.9$ & $71.6 \pm 21.7$ & $<0.001$ \\
\hline Glucose (mmol/l) & $4.5 \pm 0.5$ & $4.4 \pm 0.5$ & 0.679 \\
\hline Insulin (mU/l) & $13.9 \pm 18.0$ & $6.5 \pm 2.7$ & $<0.001$ \\
\hline HOMA-IR & $2.8 \pm 3.6$ & $1.3 \pm 0.6$ & $<0.001$ \\
\hline Pubarche (years) & $11.8 \pm 1.3$ & $11.5 \pm 1.7$ & 0.196 \\
\hline Menarche (years) & $12.1 \pm 1.7$ & $12.4 \pm 1.6$ & 0.430 \\
\hline Oligomenorrhea & $68.3 \%$ & $0 \%$ & $<0.001$ \\
\hline Amenorrhea & $22.3 \%$ & $0 \%$ & $<0.001$ \\
\hline Hirsutism & $75.2 \%$ & $11.8 \%$ & $<0.001$ \\
\hline Acne & $50.8 \%$ & $17.9 \%$ & $<0.001$ \\
\hline Total cholesterol (mmol/l) & $4.6 \pm 0.4$ & NA & NA \\
\hline HDL cholesterol $(\mathrm{mmol} / \mathrm{l})$ & $1.3 \pm 0.4$ & NA & NA \\
\hline LDL cholesterol (mmol/l) & $2.6 \pm 0.4$ & NA & NA \\
\hline Triglycerides (mmol/l) & $1.4 \pm 0.1$ & NA & NA \\
\hline CRP (mg/l) & $2.1 \pm 0.6$ & NA & NA \\
\hline
\end{tabular}

${ }^{\text {a }}$ Student's t-test was used for continuous and $\chi^{2}$ test for categorical variables. Values are expressed as the mean \pm standard deviation. PCOS, polycystic ovary syndrome; BMI, body mass index; WHR, waist/hip ratio; CAD, coronary artery disease; FSH, follicle stimulating hormone; LH, luteinizing hormone; TT, total testosterone; FT, free testosterone; DHEAS, dehydroepiandrosterone sulphate; SHBG, sex hormone binding globulin; HOMA-IR, homeostatic model assessment of insulin resistance; HDL, high-density lipoprotein; LDL, low-density lipoprotein; CRP, C-reactive protein; NA, not available.

chain reaction (PCR) using High-Fidelity Platinum DNA Polymerase (Invitrogen, Paisley, Scotland, UK), the forward primer 5'-GAACTCGAGAGGCAGAGGCAGCAGTGA-3' and the reverse primer 5'-AGAAATCACCCACTCCCT GA-3' (11), followed by electrophoresis on Spreadex Mini gels (Elchrom Scientific, Cham, Switzerland) (17). The number of TAAAA repeats was calculated from the size of the PCR products in association with the M3 marker (Elchrom Scientific) and our own internal standard. The latter was prepared from six different PCR fragments, which were 133, 138, 143, 148, 153 and 158 bp in size. Following direct sequencing, the fragments were identified as having 6-11 TAAAA repeats each. The gel was documented with ChemiGenious Bio Imaging System (Syngene, Cambridge, UK) and analyzed with GeneTool analysis software (Syngene), which allows accurate measurement of the PCR fragment length. Genotype analysis was conducted at the Institute of Medical Genetics, Department of Obstetrics and Gynecology, University Medical Centre Ljubljana (Ljubljana, Slovenia).

Statistical analysis. Values are expressed as percentages for categorical variables and as the means \pm standard deviation 
Table II. Association between different hormonal and metabolic parameters and serum SHBG levels in PCOS patients.

\begin{tabular}{lcc}
\hline & \multicolumn{2}{c}{ SHBG (nmol/l) } \\
\cline { 2 - 3 } Variables & $\mathrm{r}_{\mathrm{s}}$ & $\mathrm{P}^{\mathrm{a}}$ \\
\hline Age (years) & -0.055 & 0.603 \\
WHR & 0.108 & 0.310 \\
Insulin (mU/l) & -0.212 & 0.044 \\
Total cholesterol (mmol/l) & -0.110 & 0.301 \\
HDL cholesterol (mmol/l) & -0.113 & 0.285 \\
LDL cholesterol (mmol/l) & -0.104 & 0.326 \\
Triglycerides (mmol/l) & 0.034 & 0.752 \\
CRP (mg/l) & -0.456 & $<0.001$ \\
\hline
\end{tabular}

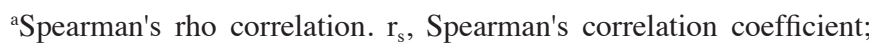
SHBG, sex hormone binding globulin; PCOS, polycystic ovary syndrome; WHR, waist/hip ratio; HDL, high-density lipoprotein; LDL, low-density lipoprotein; CRP, C-reactive protein.

for continuous variables. An independent Student's t-test was used to compare the mean values between cases and controls. Differences in categorical characteristics between cases and controls were assessed using the $\chi^{2}$ test.

For genotype analysis, the combinations of the TAAAA genotypes found in the PCOS group were clustered according to the length of alleles carried, as suggested in the study by Xita et al (16). Short-allele genotypes were defined as those having $6 / 6,6 / 7,6 / 8,7 / 7$ or $7 / 8$ repeats, and long-allele genotypes as those having $8 / 8,8 / 9,8 / 10,8 / 11,9 / 9,9 / 10,9 / 11$, or $10 / 10$ repeats. A total of 18 heterozygous combinations being one allele shorter and one allele longer than eight repeats were excluded from subsequent analyses (16).

The Spearman's correlation coefficient $\left(r_{s}\right)$ was used to assess the linear associations between different hormonal and metabolic parameters with serum SHBG levels. A Mann-Whitney U test was used to compare PCOS patients according to the length of their SHBG repeat polymorphisms, and a $\chi^{2}$ test was used for categorical characteristics.

To explore the effect of the TAAAA repeat length on serum SHBG concentration, a multiple linear regression model was constructed with SHBG as a dependent variable, and age, WHR, HOMA-IR and TAAAA allelic repeats (short/long) as independent variables.

All statistical analyses were performed using SPSS for Windows (version 15.0; SPSS, Inc., Chicago, IL, USA). $\mathrm{P}<0.05$ was considered to indicate a statistically significant difference.

\section{Results}

Clinical and biochemical characteristics of PCOS patients and controls. The clinical and hormonal parameters in PCOS patients and controls are presented in Table I. The mean age of PCOS patients was significantly lower than that in the control group $(28.3 \pm 5.78$ vs. $31.3 \pm 4.8 ; \mathrm{P}<0.001)$ As expected, LH, mean serum TT, FT and HOMA-IR were

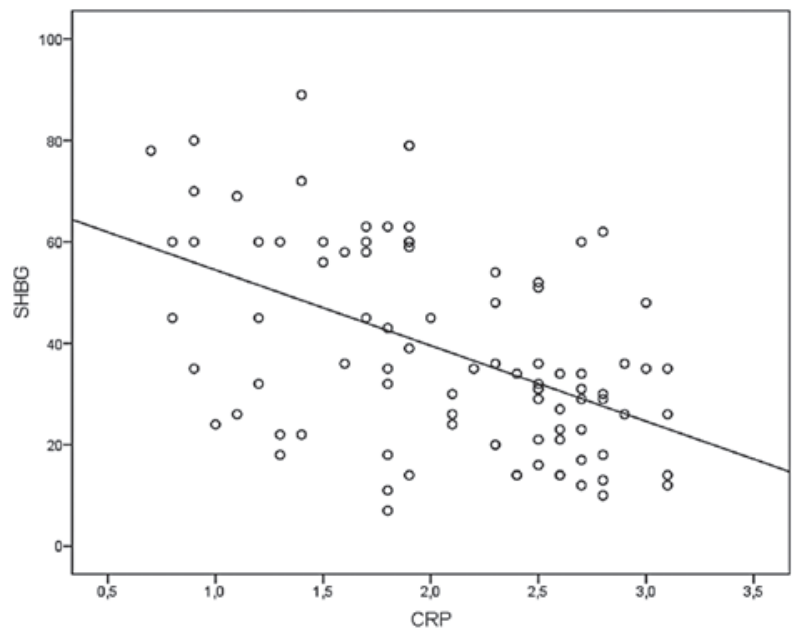

Figure 1. Association of SHBG and CRP levels in polycystic ovary syndrome patients $(\mathrm{R}=-0.456, \mathrm{P}<0.001)$. SHBG, sex hormone binding globulin; $\mathrm{CRP}$, C-reactive protein.

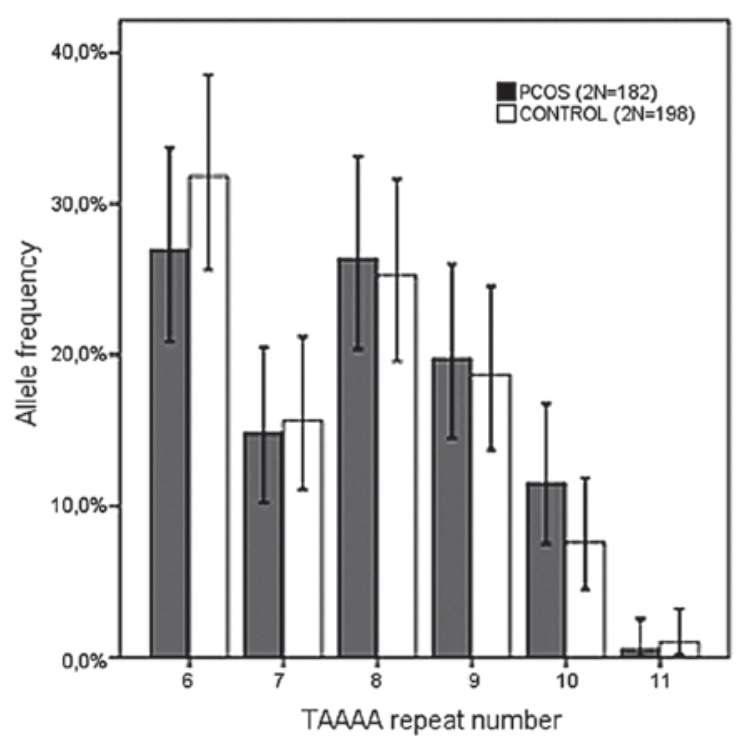

Figure 2. Distribution of alleles of the TAAAA repeat in polycystic ovary syndrome patients and controls. PCOS, polycystic ovary syndrome. The bars represent the observed frequencies for alleles with varying numbers of repeats. The distribution of alleles of the TAAAA repeat did not differ significantly between PCOS patients and healthy controls $(\mathrm{P}=0.739)$.

significantly higher in the PCOS group than those in the controls $(\mathrm{P}<0.001)$, whereas the mean serum SHBG and FSH levels were significantly lower in the PCOS group compared to those in the control group $(\mathrm{P}<0.001)$. Over $2 / 3$ (69.2\%; 63 out of 91 ) of PCOS patients were lean and $\sim 1 / 3$ reported a family history of CAD $(27.5 \% ; 25$ out of 91$)$, hypertension $(33 \% ; 30$ out of 91$)$ and diabetes $(36.3 \%$; 33 out of 91$)$. Moreover, PCOS subjects reported a higher prevalence of childhood obesity $(42.9 \%$; 39 out of 91$)$ compared to adult obesity $(30.8 \%$; 28 out of 91) (Table I).

Association between different hormonal and metabolic parameters and serum SHBG levels in patients with PCOS. A weak yet significant negative correlation was observed between serum SHBG and insulin levels $\left(r_{s}=-0.212, P=0.044\right)$. 
Table III. Clinical, biochemical and cardiovascular risk parameters of patients with polycystic ovary syndrome according to the length of the SHBG repeat polymorphism.

\begin{tabular}{|c|c|c|c|}
\hline Variable & $\begin{array}{l}\text { Short } \\
(n=38)\end{array}$ & $\begin{array}{l}\text { Long } \\
(n=35)\end{array}$ & P-value ${ }^{a}$ \\
\hline Age (years) & $28.6 \pm 6.6$ & $28.4 \pm 5.1$ & 0.740 \\
\hline BMI $\left(\mathrm{kg} / \mathrm{m}^{2}\right)$ & $24.0 \pm 4.5$ & $24.3 \pm 4.9$ & 0.667 \\
\hline $\mathrm{BMI}<25\left(\mathrm{~kg} / \mathrm{m}^{2}\right)$ & $76.3 \%$ & $64.7 \%$ & 0.279 \\
\hline WHR & $0.79 \pm 0.1$ & $0.78 \pm 0.1$ & 0.916 \\
\hline Family history of CAD & $26.3 \%$ & $28.6 \%$ & 0.829 \\
\hline Hypertension in family & $26.3 \%$ & $37.1 \%$ & 0.320 \\
\hline Dyslipidemia in family & $0 \%$ & $11.4 \%$ & 0.032 \\
\hline Diabetes in family & $36.8 \%$ & $40.0 \%$ & 0.782 \\
\hline History of hypertension & $2.6 \%$ & $2.9 \%$ & 0.953 \\
\hline Childhood obesity & $50.0 \%$ & $42.9 \%$ & 0.541 \\
\hline FSH (IU/l) & $3.2 \pm 0.9$ & $3.3 \pm 0.7$ & 0.504 \\
\hline LH (IU/l) & $7.9 \pm 6.2$ & $8.3 \pm 4.6$ & 0.126 \\
\hline TT (nmol/l) & $2.0 \pm 1.0$ & $1.9 \pm 0.7$ & 0.489 \\
\hline FT (pmol/l) & $31.2 \pm 21.1$ & $47.2 \pm 24.2$ & 0.001 \\
\hline $\mathrm{AN}(\mathrm{nmol} / \mathrm{l})$ & $10.9 \pm 5.8$ & $12.1 \pm 4.4$ & 0.090 \\
\hline DHEAS $(\mu \mathrm{mol} / \mathrm{l})$ & $6.1 \pm 3.2$ & $7.3 \pm 2.5$ & 0.051 \\
\hline SHBG (nmol/l) & $50.9 \pm 19.4$ & $24.1 \pm 11.3$ & $<0.001$ \\
\hline Glucose (mmol/l) & $4.4 \pm 0.4$ & $4.5 \pm 0.4$ & 0.465 \\
\hline Insulin (mU/l) & $15.2 \pm 23.5$ & $11.5 \pm 7.9$ & 0.991 \\
\hline HOMA-IR & $2.9 \pm 4.2$ & $2.3 \pm 1.6$ & 0.912 \\
\hline Total cholesterol (mmol/l) & $4.5 \pm 0.4$ & $4.6 \pm 0.3$ & 0.099 \\
\hline HDL cholesterol (mmol/l) & $1.4 \pm 0.4$ & $1.5 \pm 0.4$ & 0.657 \\
\hline LDL cholesterol (mmol/l) & $2.5 \pm 0.5$ & $2.7 \pm 0.3$ & 0.049 \\
\hline Triglycerides (mmol/l) & $1.4 \pm 0.2$ & $1.3 \pm 0.1$ & 0.407 \\
\hline $\mathrm{CRP}(\mathrm{mg} / \mathrm{l})$ & $1.7 \pm 0.6$ & $2.4 \pm 0.4$ & $<0.001$ \\
\hline Age at pubarche (years) & $11.3 \pm 1.6$ & $12.8 \pm 1.3$ & 0.731 \\
\hline Age at menarche (years) & $12.8 \pm 1.4$ & $12.7 \pm 1.9$ & 0.479 \\
\hline
\end{tabular}

${ }^{a}$ The Mann-Whitney $U$ test was used for continuous and $\chi^{2}$ test for categorical variables. Values are expressed as the mean \pm standard deviation. BMI, body mass index; WHR, waist/hip ratio; CAD, coronary artery disease; FSH, follicle stimulating hormone; $\mathrm{LH}$, luteinizing hormone; TT, total testosterone; FT, free testosterone; AN, androstenedione; DHEAS, dehydroepiandrosterone sulphate; SHBG, sex hormone binding globulin; HOMA-IR, homeostatic model assessment of insulin resistance; HDL, high-density lipoprotein; LDL, low-density lipoprotein; CRP, C-reactive protein.

Serum SHBG levels were found to be inversely correlated with serum CRP levels $\left(r_{s}=-0.456, P<0.001\right)$ (Fig. 1) in PCOS patients. The negative correlation remained significant following adjustment for BMI, WHR, TT, insulin, total cholesterol, LDL and triglyceride levels $(\mathrm{R}=-0.477, \mathrm{P}<0.001)$. No significant linear associations were found between the other cardiovascular factors evaluated in the PCOS group (Table II).

Association between the TAAAA repeat polymorphism and $P C O S$. The distribution of alleles of the TAAAA repeat
Table IV. Multiple linear regression models with sex hormone binding globulin as the dependent variable.

\begin{tabular}{llr}
\hline Variable & B-coefficient (95\% CI) & P-value \\
\hline $\begin{array}{l}\text { Unadjusted model } \\
\left(R^{2}=0.417\right)\end{array}$ & & \\
TAAAA repeat length & -25.637 (from -32.813 & $<0.001$ \\
& to -18.461$)$ & \\
Adjusted model & & \\
$\left(R^{2}=0.448\right)$ & & \\
TAAAA repeat length & -26.876 (from -34.377 & $<0.001$ \\
& to -19.376$)$ & 0.135 \\
WHR & -34.370 (from -79.653 & \\
HOMA-IR & to 10.913$)$ & 0.954 \\
& 0.034 (from -1.130 & \\
Age (years) & to 1.197$)$ & \\
& -0.449 (from -1.090 & \\
& to 0.191 ) &
\end{tabular}

CI, confidence interval; WHR, waist to hip ratio; HOMA-IR, homeostatic model assessment of insulin resistance.

polymorphism is presented in Fig. 2. The highest frequency was observed for alleles with six $(26.9 \%$ in PCOS vs. $31.8 \%$ in control group), eight (26.4\% in PCOS vs. $25.3 \%$ in control group) and nine repeats (19.8\% in PCOS vs. $18.7 \%$ in control group). The distribution of alleles of the TAAAA repeat did not differ significantly between PCOS patients and healthy controls $(\mathrm{P}=0.739)$.

Association between the TAAAA repeat polymorphism and the clinical, biochemical and cardiovascular risk parameters in PCOS patients. The correlation of clinical, biochemical and cardiovascular risk parameters with the length of TAAAA repeats was assessed. The PCOS patients $(47.9 \%$; 35 out of 73$)$ with long TAAAA alleles $(8 / 8,8 / 9,8 / 10$, $8 / 11,9 / 9,9 / 10,9 / 11$ or $10 / 10$ repeats) had significantly lower serum SHBG levels $(\mathrm{P}<0.001)$ and significantly higher FT $(\mathrm{P}=0.001)$ and CRP levels $(\mathrm{P}<0.001)$ than those of the PCOS patients $(52.1 \%$; 38 out of 73$)$ with short alleles $(6 / 6,6 / 7,6 / 8$, $7 / 7$ or $7 / 8$ repeats) (Table III). A family history of dyslipidemia was only present in the long allele group; however, no significant difference was observed for other anthropometric or biochemical parameters between the groups (Table II). No difference was observed between the age at pubarche and menarche according to the genotype length (Table III).

To explore the association between the SHBG repeat length and SHBG levels, multivariate analysis was performed with SHBG as a dependent variable. The TAAAA allelic repeat (short/long) and various other factors that may influence SHBG levels (WHR, HOMA-IR, age) were included as independent variables. This model revealed a significant influence of the longer TAAAA genotype on lower serum SHBG levels in patients with PCOS $(\mathrm{P}<0.001)$ (Table IV). 


\section{Discussion}

In the present study, the influence of the pentanucleotide polymorphism in the SHBG coding gene on the circulating SHBG levels was evaluated in 91 Croatian females with PCOS and 99 healthy controls. Additionally, the influence of low SHBG levels on early cardiovascular risk factors in patients with PCOS patients was assessed.

To the best of our knowledge, the present study was the first to examine the association between the TAAAA SHBG repeat length and PCOS in the Croatian population. The frequency and distribution of the TAAAA alleles discovered were comparable to those reported earlier in other PCOS populations (16-19). The TAAAA alleles were equally distributed between the PCOS and the control group, indicating that this type of polymorphism is not the major determinant of PCOS.

In the present study, PCOS patients with long TAAAA alleles had significantly lower SHBG levels compared with those carrying short alleles. The observed association was confirmed in the linear regression model that included other factors known to influence SHBG levels (WHR, HOMA-IR, age). As expected, the results revealed significantly higher serum free testosterone levels in the PCOS group carrying long alleles compared with those of the short allele group. A possible genetic contribution of this polymorphism to the decreased serum SHBG levels has been reported in females with PCOS from different ethnic backgrounds $(14,16-18)$. It has been suggested that these females may have been exposed to higher androgen levels during their foetal development, which may have programmed their PCOS phenotype (14,25).

It has been proposed that TAAAA repeat alleles may be associated with early menarche - a known risk factor for the development of PCOS (20); however, this was not observed in the present study. The results of the present study did not reveal any significant differences in the age at pubarche or menarche between PCOS patients with long and those with short TAAAA alleles. The fact that the information on age at pubarche and menarche was self-reported may have biased these results. In addition, the frequency of other known risk factors for PCOS (low birth weight, preterm birth and childhood obesity) were compared between PCOS patients with short and long allele repeats, but no significant difference was observed. Data for the control group were unavailable.

Additionally, the influence of low SHBG levels on different cardiovascular risk factors was assessed in patients with PCOS. Low SHBG levels have been proposed as independent predictors for the development of cardiovascular disease (2,10-13) and metabolic syndrome (8) in males and females.

In a cross-sectional study recently published by Liao et al (11), an independent negative correlation was observed between SHBG and CRP in males, as well as premenopausal and postmenopausal females. In the present study, a strong negative linear association was observed between serum SHBG and CRP levels, even following adjustment for BMI, WHR, TT, HOMA-IR, total cholesterol, LDL cholesterol and triglyceride levels. No other association between serum SHBG levels and cardiovascular risk factors was found. This may be due to the low incidence of cardiovascular risk parameters (BMI $>25 \mathrm{~kg} / \mathrm{m}^{2}$, insulin resistance and metabolic syndrome) within the study population. Previous results by our group have shown that the Croatian patients with PCOS have a low prevalence of obesity ( $<1 / 3$ of patients) and abdominal accumulation of fat, and that the most prominent findings are hyperandrogenemia and hyperandrogenism, which were present in $>2 / 3$ of PCOS patients (26). Consequently, the independent negative association between SHBG and CRP that was observed in the present study may be the result of continuous exposure to higher free androgen levels due to reduced levels of SHBG, although the present cross sectional study on a small number of patients with PCOS and healthy controls had limited power to assess the influence of an excess of androgens on cardiovascular risk. Therefore, the influence of excess androgen on metabolic disorders and cardiovascular risk in females remains to be a matter of debate. Recent studies have shown a strong association between hyperandrogenism and inflammation in PCOS (27-30). González et al (30) have recently reported that leukocytes of lean reproductive-age females generate a pro-oxidant response following raising circulating androgens to levels present in PCOS, which supports the concept that in PCOS, hyperandrogenism may be a progenitor of diet-induced oxidative stress, independent of obesity or excess abdominal adiposity (31). Oxidative stress causes lipid peroxidation, protein carbonylation and DNA damage (32-34). This in turn activates nuclear factor $\kappa \mathrm{B}(\mathrm{NF \kappa B})$ to stimulate the transcription of tumor necrosis factor $\alpha(\mathrm{TNF} \alpha)(27-29)$, which in turn promotes the synthesis of interleukin (IL)-6 (35). IL-6 stimulates the liver and adipose tissue production of CRP, which promotes the uptake of lipids into foamy macrophages within atherosclerotic plaques (36). In line with this finding, it has been recently reported that sustained exposure to elevated levels of TNFa decreases SHBG production by reducing hepatic hepatocyte nuclear factor- $4 \alpha$ levels via NF- $\kappa B$ activation in human HepG2 hepatoblastoma cells (37).

The present study has shown that in a Croatian cohort with a low prevalence of obesity, low plasma SHBG levels are significantly associated with high levels of CRP. This significant association between SHBG and high CRP was independent of insulin resistance measured by HOMA-IR, supporting low SHBG levels as an independent marker of enhanced cardiovascular risk in females.

Since a strong association between serum SHBG levels and the number of TAAAA repeat alleles is well established (14,16-18), the present study evaluated the differences in various cardiovascular risk parameters between PCOS patients with short and long repeats. The serum CRP levels were significantly higher in patients with long TAAAA allele repeats compared with those of patients with short repeats. A significant difference between other parameters evaluated was not observed, except for a family history of dyslipidemia, which was found only in the long allele group. However, the overall incidence of dyslipidemia was too low to reach any conclusions. Alveziaki et al (20) reported that longer TAAAA repeat alleles are associated with more severe coronary artery disease in postmenopausal females. Saltiki et al (21) reported that longer TAAAA repeats in the SHBG gene promoter are associated with impaired endothelial function, an early marker of atherosclerosis in healthy females. The results of the present study provided further evidence that TAAAA repeat polymorphism of the SHBG gene is associated with higher CRP levels. CRP is an established risk factor of CVD and a marker of low-grade 
inflammation, typical of atherogenesis. This may be one of the pathways by which low SHBG levels affect cardiovascular risk.

In conclusion, TAAAA repeat polymorphism of the SHBG gene promoter is not a major determinant of the PCOS status; however, it influences serum SHBG levels in females with PCOS. A strong inverse independent correlation exists between serum SHBG and CRP levels. The exact causal association between the genetically decreased serum SHBG levels and the expression of the PCOS phenotype and metabolic abnormalities accompanying the syndrome require further testing in larger population studies.

\section{Acknowledgements}

This study was supported by a grant from the Ministry of Science, Education and Sports of the Republic of Croatia (no. 108-0000000-0388).

\section{References}

1. Rotterdam ESHRE/ASRM-Sponsored PCOS consensus workshop group: Revised 2003 consensus on diagnostic criteria and long-term health risks related to polycystic ovary syndrome (PCOS). Hum Reprod 19: 41-47, 2004.

2. Rotterdam ESHRE/ASRM-Sponsored PCOS Consensus Workshop Group: Revised 2003 consensus on diagnostic criteria and long-term health risks related to polycystic ovary syndrome. Fertil Steril 81: 19-25, 2004.

3. Carmina E, Chu MC, Longo RA, Rini GB and Lobo RA: Phenotypic variation in hyperandrogenic women influences the finding of abnormal metabolic and cardiovascular risk parameters. J Clin Endocrinol Metab 90: 2545-2549, 2005.

4. Boulman N, Levy Y, Leiba LR, Shachar S, et al: Increased C-reactive protein levels in polycystic ovary syndrome: a maker of cardiovascular disease. J Clin Endocrinol Metab 89: 2160-2165, 2004.

5. Morin-Papunen L, Rautio K, Ruokonen A, Hedberg P, Puukka M and Tapanainen JS: Metformin reduces serum C-reactive protein levels in women with polycystic ovary syndrome. J Clin Endocrinol Metab 88: 4649-4654, 2003.

6. Escobar-Morreale HF,Luque-Ramírez M and González F: Circulating inflammatory markers in polycystic ovary syndrome: a systematic review and metaanalysis. Fertil Steril 95: 1048-1058, 2011.

7. Toulis KA, Goulis DG, Mintziori G, et al: Meta-analysis of cardiovascular disease risk markers in women with polycystic ovary syndrome. Hum Reprod Update 17: 741-760, 2011.

8. Brand JS, van der Tweel I, Grobbee DE, Emmelot-Vonk MH and van der Schouw YT: Testosterone, sex hormone-binding globulin and the metabolic syndrome: a systematic review and meta-analysis of observational studies. Int J Epidemiol 40: 189-207, 2011.

9. Speroff L, Glass RH and Kase NG (eds): Anovulation and the polycystic ovary. In: Clinical Gynecologic Endocrinology and Infertility. Lippincott Wiliams \& Wilkins, Philadelphia, USA, pp487-521, 1999.

10. Peter A, Kantartzis K, Machann J, et al: Relationships of circulating sex hormone-binding globulin with metabolic traits in humans. Diabetes 59: 3167-3173, 2010.

11. Liao CH, Li HY, Yu HJ, et al: Low serum sex hormone-binding globulin: marker of inflammation? Clin Chim Acta 413: 803-807, 2012.

12. Bonnet F, Balau B, Malécot JM, et al: Sex hormone-binding globulin predicts the incidence of hyperglycemia in women: interactions with adiponectin levels. Eur J Endocrinol 161: 81-85, 2009.

13. Ding EL, Song Y, Manson JE, et al: Sex hormone-binding globulin and risk of type 2 diabetes in women and men. N Engl J Med 361: $1152-1163,2009$

14. Xita $\mathrm{N}$ and Tsatsoulis A: Genetic variants of sex hormone-binding globulin and their biological consequences. Mol Cell Endocrinol 316: 60-65, 2010.

15. Hogeveen KN, Talikka M and Hammond GL: Human sex hormone-binding globulin promoter activity is influenced by a (TAAAA)n repeat element within an Alu sequence. J Biol Chem 276: 36383-36390, 2001.
16. Xita N, Tsatsoulis A, Chatzikyriakidou A and Georgiou I: Association of the (TAAAA)n repeat polymorphism in the sex hormone-binding globulin (SHBG) gene with polycystic ovary syndrome and relation to SHBG serum levels. J Clin Endocrinol Metab 88: 5976-5980, 2003.

17. Ferk P, Teran N and Gersak K: The (TAAAA)n microsatellite polymorphism in the SHBG gene influences serum SHBG levels in women with polycystic ovary syndrome. Hum Reprod 22: 1031-1036, 2007.

18. Cousin P, Calemard-Michel L, Lejeune H, et al: Influence of SHBG gene pentanucleotide TAAAA repeat and D327N polymorphism on serum sex hormone-binding globulin concentration in hirsute women. J Clin Endocrinol Metab 89: 917-924, 2004.

19. Liu Q, Gu W, Cui B, et al: The association of TAAAAn repeat polymorphism in sex hormone-binding protein gene with polycystic ovary syndrome in Chinese population. Endocrine 34 : 62-67, 2008.

20. Alevizaki M, Saltiki K, Xita N, et al: The importance of the (TAAAA)n alleles at the SHBG gene promoter for the severity of coronary artery disease in postmenopausal women. Menopause 15: 461-468, 2008.

21. Saltiki K, Stamatelopoulos K, Voidonikola P, et al: Association of the SHBG gene promoter polymorphism with early markers of atherosclerosis in apparently healthy women. Atherosclerosis 219 : 205-210, 2011.

22. Ferriman D and Gallwey JD: Clinical assessment of body hair growth in women. J Clin Endocrinol Metab 21: 1440-1447, 1961.

23. Vermeulen A, Verdonck L and Kaufman JM: A critical evaluation of simple methods for the estimation of free testosterone in serum. J Clin Endocrinol Metab 84: 3666-3672, 1999.

24. Friedewald WT, Levy RI and Fredericson DS: Estimation of the concentration of low density lipoprotein cholesterol without use of the ultracentrifuge. Clin Chem 18: 499-502, 1972.

25. Tsatsoulis A: Developmental programming of polycystic ovary syndrome: role of prenatal androgen excess. In: Tsatsoulis A, Wyckoff J, Brown FM (eds) Diabetes in Women: Pathophysiology and Therapy, Humana Press, New York, NY, pp83-103, 2009.

26. Baldani DP, Skrgatić L, Goldstajn MS et al: Clinical and biochemical characteristics of polycystic ovary syndrome in Croatian population. Coll Antropol 36: 1413-1418, 2012.

27. González F, Rote NS, Minium J and Kirwan JP: Increased activation of nuclear factor kappaB triggers inflammation and insulin resistance in polycystic ovary syndrome. J Clin Endocrinol Metab 91: 1508-1512, 2006.

28. González F, Rote NS, Minium J and Kirwan JP: Reactive oxygen species-induced oxidative stress in the development of insulin resistance and hyperandrogenism in polycystic ovary syndrome. J Clin Endocrinol Metab 91: 336-340, 2006.

29. González F, Rote NS, Minium J and Kirwan JP: Evidence of proatherogenic inflammation in polycystic ovary syndrome. Metabolism 58: 954-962, 2009.

30. González F, Nair KS, Daniels JK, et al: Hyperandrogenism sensitizes leukocytes to hyperglycemia to promote oxidative stress in lean reproductive-age women. J Clin Endocrinol Metab 97: 2836-2843, 2012.

31. González F: Inflammation in polycystic ovary syndrome: underpinning of insulin resistance and ovarian dysfunction. Steroids 77: 300-305, 2012.

32. Jain SK: Hyperglicemia can cause membrane lipid peroxidation and osmotic fragility in human red blood cells. J Biol Chem 264: 21340-21345, 1989.

33. Aljada A, Thusu K, Amstrong D, Nicotera T and Dandona P: Increased carbonylation of proteins in diabetes. Diabetes 44: 113A, 1995

34. Dandona P, Thusu K, Cook S, et al: Oxidative damage to DNA in diabetes mellitus. Lancet 347: 444-445, 1996.

35. Stephens JM, Butts MD and Pekala PH: Regulation of transcription factor mRNA accumulation during 3T3-L1 preadipocytedifferentiation by tumour necrosis factor-alpha. J Mol Endocrinol 9: 61-72, 1992.

36. Zwaka TP, Hombach V and Torzewski J: C-reactive protein-mediated low density lipoprotein uptake by macrophages: implications for atherosclerosis. Circulation 103: 1194-1197, 2001.

37. Simó R, Barbosa-Desongles A, Sáez-Lopez C, et al: Molecular mechanism of $\mathrm{TNF} \alpha$-induced down-regulation of SHBG expression. Mol Endocrinol 26: 438-446, 2012. 